\title{
Multiscale modelling for better hygrothermal prediction of porous building materials
}

\author{
Rafik BELARBI ${ }^{1}$, Fares BENNAI ${ }^{1,2}$, MohammedYacine FERROUKHI ${ }^{3}$, ChadyEL HACHEM ${ }^{2}$, Kamilia ABAHRI ${ }^{2}$ \\ ${ }^{1}$ Université de La Rochelle - CNRS, LaSIE UMR 7356, Avenue Michel Crépeau 17042 La Rochelle Cedex 1, France \\ ${ }^{2}$ LMT, ENS-Cachan, CNRS, Université Paris Saclay, 61 avenue du Président Wilson, F-94230 Cachan, France \\ ${ }^{3}$ ENISE, Université de Lyon, LTDS CNRS UMR 5513, 58 Avenue Jean Parot, 42023 Saint Etienne, France
}

\begin{abstract}
The aim of this work is to understand the influence of the microstructuralgeometric parameters of porous building materials on the mechanisms of coupled heat, air and moisture transfers, in order to predict behavior of the building to control and improve it in its durability. For this a multi-scale approach is implemented. It consists of mastering the dominant physical phenomena and their interactions on the microscopic scale. Followed by a dual-scale modelling, microscopic -macroscopic, of coupled heat, air and moisture transfers that takes into account the intrinsic properties and microstructural topology of the material using X-ray tomography combined with the correlation of 3D images were undertaken. In fact, the hygromorphicbehavior under hydric solicitations was considered. In this context, a model of coupled heat, air and moisture transfer in porous building materials was developed using the periodic homogenization technique. These informations were subsequently implemented in a dynamic computation simulation that model the hygrothermalbehaviourof material at the scale of the envelopes and indoor air quality of building. Results reveals that is essential to consider the local behaviors of materials, but also to be able to measure and quantify the evolution of its properties on a macroscopic scale from the youngest age of the material. In addition, comparisons between experimental and numerical temperature and relative humidity profilesin multilayers wall and in building envelopes were undertaken. Good agreements were observed.
\end{abstract}

\section{Introduction}

Nowadays, the study of coupled heat and mass transfers in porous building materials becomes an essential part of the methodological arsenal of the modern thermal studies. It became essential step to corroborate the energetic and environmental challenges. In fact, coupled heat air and mass transfers at the scale of porous materials and that ofthe building-environment interface are complex in many respects. It is multi-scale (pore, material, envelope and whole building) and multiphysical (mass transfer, heat with strong interaction). One of the major challenges is to identify the most influential parameters defining the overall behavior of the building (new and old) and the interactions that exist between subsystems in order to propose a design adapted to the built spaces. The knowledge of such a response is important both in terms of predicting the quality of habitable environments and assessing the durability of structures [1].

The preliminary choice of materials used for construction today presents an alternative solution to meet its problems. This certainly reduces the energy and environmental impact, but reveals new challenges, as this could alter summer comfort and indoor air quality.
In addition, the use of materials with low environmental impact, such as bio-based mortars, already known for their thermal insulation properties, acoustic properties and low environmental impact, is increasingly taking place in the construction industry last year's [2].

Moreover, the use of these materials is hampered, in particular, by the unavailability of databases relating to their intrinsic properties and by ignorance of their behaviors over time. This use requires taking into account several criteria that make them vulnerable; the problem of swelling-shrinkage at the cement-fiber interface and fungal growth are the most important criteria for assessing the degradation of these materials.

Indeed, few works in the literature, in the field of civil engineering, take into consideration the effect of local material behavior when modelling the envelope scale ...

Indeed, the macroscopic behavior of these materials is highly dependent on the mechanisms involved on a microscopic scale. In order to predict the behavior of structures on a macroscopic scale, it is essential to know how to capture local behaviors, but also to be able to measure and quantify the evolution of these properties on a macroscopic scale from the youngest age of the 
material. At the scale of the pore, the physical transfer processes and the topology of the liquid interfaces (bound or free) vapor, air and their interactions with the solid matrix (contact lines, detachment of vapor bubbles from solid surfaces, capillary bridges, ...) make the study of these processes extremely complex. In particular, it is necessary to link the phenomena of mechanical deformations (intra-material swelling and capillary pressure / disjunction) to the equilibrium states of moisture in materials [3].

That why, the present study made recourse to nondestructive method to made refine morphological characterization of the main intrinsic properties of the studied materials. This first step will provide information on the three-dimensional microstructure and also allow to locate any heterogeneity, singularity, porosity, specific surface area and to deduce the representative structure and its deviations via $3 \mathrm{D}$ reconstruction techniques.

Then, the implementation of homogenised approachesusing 3D numerical modelling on the real structures of the materials provides more information about the equivalent macroscopic behaviour of the material. These information at the material scale will be then implemented when modelling the envelopes responses to the different hygrothermal solicitations representatives the building environment.

\section{Microscopic characterization}

\section{$2.13 \mathrm{D}$ reconstruction}

The recourse tonon-destructive methods presents some assets for a better microstructural characterization at the local scale of the material.

$\mathrm{X}$-ray tomography is one of these non-destructive imaging techniques that consists in reconstructing the $3 \mathrm{D}$ volume of an object from a series of 2D x-rays. This technique was developed and used primarily in the medical field, then in the industry of the director of nondestructive testing and making dimensional measurements. The resolution of tomographic images is function of many parameters, like the distance between the source and the specimen. it is directly related to the dimensions of the specimen.

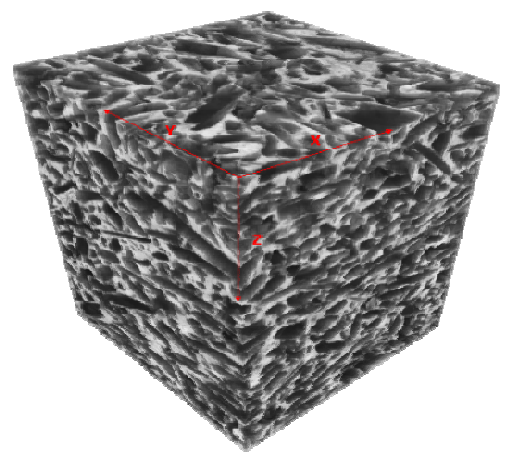

Fig. 1.3D reconstruction of hemp concrete at a resolution of $31.8 \mu \mathrm{m}$

The studiedmaterial is hemp concrete, which consists of hemp shiv, water and binder (mixture of: natural lime from Saint-Astier (Hydraulic: NHL and aerial: CL), cement CEM I 52.5, and different adjuvants to improve the rheology and permeability of hemp concretes).

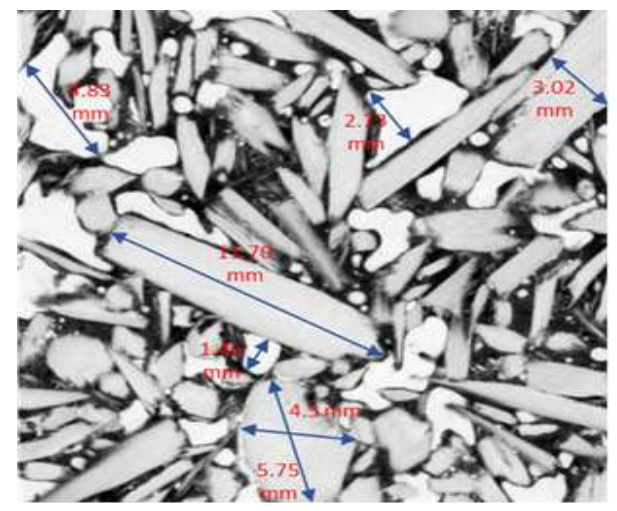

Fig. 2.2D section of a volume of hemp concrete scanned at $31.8 \mu \mathrm{m}$.

Considering that the material contains hemp shives which are weakly absorbing material, a special treatment was required to perform imaging scans. After performing and analyzing many tests on this material in the tomograph, a choice of using voltage of $100 \mathrm{kV}$ was selected for all the tests treated in this study.

Fig. 2 shows a macro porosity up to a few millimeters in size and random distribution of the hemps shiv. This macro porosity is related to the arrangements of plant particles between them.

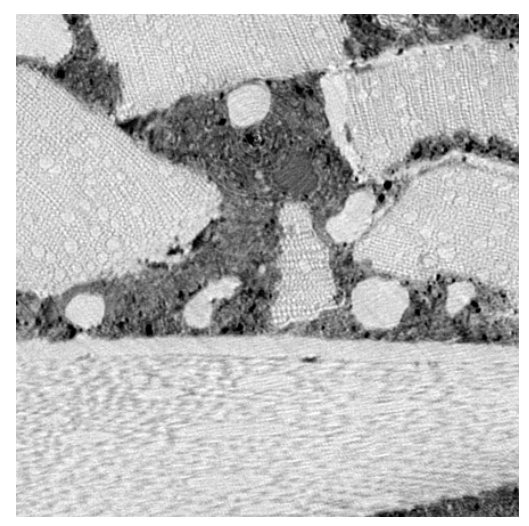

Fig. 3. 2D section of a volume of hemp concrete scanned at $2.3 \mu \mathrm{m}$.

Fig. 3 shows an image of the hemp concrete scanned at $2.3 \mu \mathrm{m}$, where a part of the porosity of hemp shives is observed. the comparison of this figure with the previous figure (Fig. 3) shows that, this very heterogeneous material exhibits a wide distribution of the pore size ranging from the micropores (at the binder and hemp shives) to the macro pores (interparticle porosity). This technique of tomography thus makes it possible to gain access to the heart of the material and to inform on the interface problems in the materialand its complex microstructure. In fact, the hemp shiv is covered by the binder leaving an interparticle porosity between the vegetable particles and the binder.it should be emphasized that the interparticle porosity contributes strongly to the improvement of the hygrothermal properties of the material by natural phase change. 


\section{2 local properties}

It was improved that the local properties are highly dependent on the material morphology especially the fibers orientation. The example cited here concerns the porosity, where the evaluation of the porosity was carried out on a sample of hemp concrete scanned with X-ray tomography at a resolution of $7.5 \mu \mathrm{m}$.

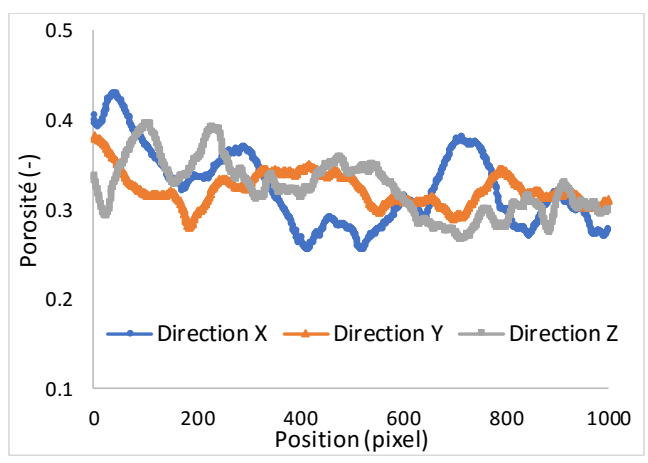

Fig. 4.Distribution of the air volume of the hemp concrete sample in the 3 directions $(\mathrm{x}, \mathrm{y}, \mathrm{z})$.

A significant heterogeneity of the calculated porosity is observed in Fig. 4, the latter exhibits fluctuations and varies as a function of the position. Indeed, the macro porosity between the binder and hemp shives depends strongly on the orientation and the position of the vegetable particles.

For this, these variabilities were seriously considered when modeling the hygrothamal transfers of such heterogenous material, for a better prediction of the behavior of the materials used in the building envelope.

\section{3 hygromorphicbehavior}

This material consists of the hemp shives and the binder, which exhibit different behaviors with respect to variations in environmental conditions, capable of causing considerable dimensional changes in contact with the relative humidity of the air. On the microscopic scale, the local deformations are due to the absorption of water by the cell walls of the hemp shiv.

The test consists of scanning the same sample of hemp concrete in the dry state ( $0 \% \mathrm{HR})$ and then in the wet state ( $85 \% \mathrm{HR})$.For this purpose, an original device adapted to the tomograph, in order to preserve a constant relative humidity of the samples during the scans.

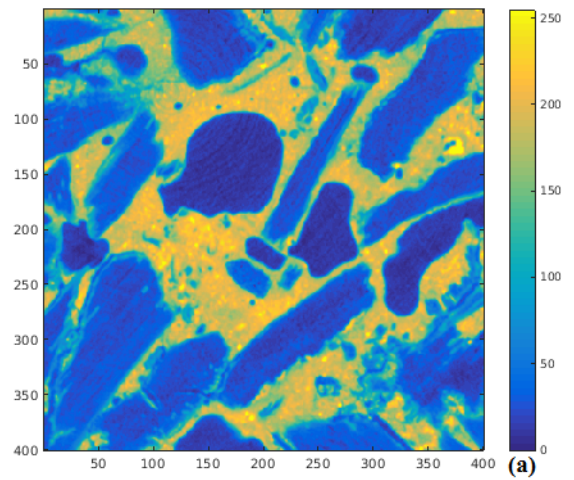

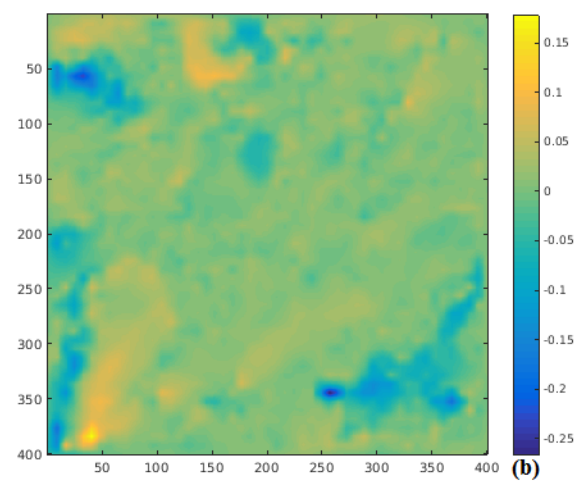

Fig. 5. (a)Section in the reference volume, (b)Deformations field $\varepsilon_{z z}(\%)$

The volume images correlation allowed to quantify the deformation fields of the studied volumes using Correli-C8R [4]. The comparison between the reference and deformed images illustrated in Fig. 5 shows that the hemp shives undergo swelling due to the phenomenon of water adsorption. This is explained by the yellow dilated zones. This swelling then contributes to the reduction of the porous space which is reflected by the blue narrowed areas.

\section{Periodic homogenization}

\section{1 developed model}

A refine periodic homogenization was developed in a previous work in order to predict the hygrothermal behavior of such material. The equations of the mass balance and of thermal equilibrium have been homogenized [5, 6]. Macroscopic descriptions of the different phenomena have been obtained. The homogenized mathematical model for coupled heat, air and moisture transfer in porous building materials can be written as follows:

$\frac{\partial \theta_{l}}{\partial t}=\operatorname{div}_{x}\left(D_{\theta} \nabla_{x} \theta_{l}+D_{T} \nabla_{x} T-D_{P} \nabla_{x} P\right)$

$C^{h o m} \frac{\partial T^{0}}{\partial t}=\operatorname{div}_{x}\left(a_{t} \nabla_{x} T+\delta_{t} \nabla_{x} \theta_{l}+\alpha_{t} \nabla_{x} P\right)-h_{l v} m_{g}$

$h_{a} \frac{\partial P}{\partial t}=\operatorname{div}_{x}\left(K_{P, P} \nabla_{x} P+K_{\theta, P} \nabla_{x} \theta_{l}+K_{T, P} \nabla_{x} T\right)-m_{g}$

This model allows us to enfranchise the assumptions of the constancy of the gas pressure and the nonisothermal conditions; as well as take into account the complexity of the internal morphology of the materials. In added, the main physical parameters of this model are non-linear and depend on the water content, temperature, and microstructure of the porous medium.

\section{2 case study}

Consider a porous material whose microstructure consists of a periodic repetition of a three-dimensional unitary cell (Fig. 6). This cell has been chosen for the purpose of numerically determining the homogenized 
diffusion and thermal conductivity tensors constituting the main input parameters of the developed model. The dimensions of this cell are chosen so as to obtain a low porosity corresponding to the porosity of the cementitious materials, such as concrete

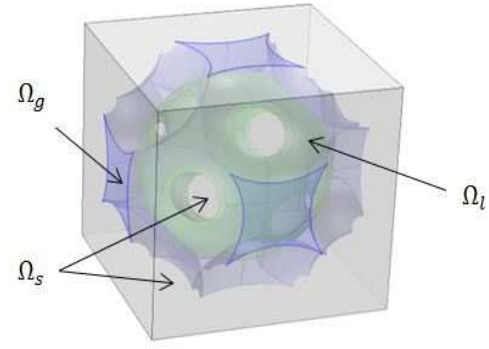

Fig. 6. Elementary cell of periodic medium

- Thermal conductivity tensor homogenized

$$
\begin{aligned}
\lambda^{\text {hom }}=\lambda_{s}\left[\begin{array}{ccc}
0.78217 & 0 & 0 \\
0 & 0.78217 & 0 \\
0 & 0 & 0.78217
\end{array}\right] & \\
& +\lambda_{l}\left[\begin{array}{ccc}
0.02188 & 0 & 0 \\
0 & 0.02188 & 0 \\
0 & 0 & 0.02188
\end{array}\right]
\end{aligned}
$$

- Diffusion tensor homogenized

$$
D_{v}^{\text {hom }}=D_{v}\left[\begin{array}{ccc}
0.04127 & 0 & 0 \\
0 & 0.04127 & 0 \\
0 & 0 & 0.04127
\end{array}\right]
$$

The results showed that these parameters depend strongly on the geometry of the cell. In addition, the experimental validation of the macroscopic diffusion coefficient was carried out in relation to the literature, and the thermal conductivity of the solid phase is thus deduced. This latter is considerably greater than the overall conductivity of the material.

Table1. Conductivité thermique de la matrice solide etcomparaison du coefficient de diffusion

\begin{tabular}{|c|c|c|c|}
\hline$\lambda_{s}(W / m . K)$ & $D_{\theta}\left(m^{2} / s\right)$ & $D_{\theta}^{\text {exp }}\left(\mathrm{m}^{2} / s\right)$ & $D_{\theta} / D_{\theta}^{\exp }$ \\
\hline 1.9135 & $2.50710^{-10}$ & $1.0110^{-10}$ & 2.47 \\
\hline
\end{tabular}

\section{Big scale modelling}

\subsection{Heat, air and moisture transfer in the envelope}

To accurately predict the hygrothermal behavior at the building envelope, we opted for a model based on Luikov theory [7, 8]. The developed model considers as driving potentials the temperature for heat transfer, the total pressure for air transfer and the water vapor pressure for the hydric transfer. This allows the avoidance of discontinuity problems at the wall layer interfaces, which is not the case with the water content.

The HAM model is built on heat and mass conservation laws. The first two equationsrepresent bothmoisture (liquid and water vapor) and gaseous balances, and the last equations expresses the heat balance by taking into account the heat conduction and heat advection due to moisture and total pressure gradients. It is noted that the capillary liquid transfer expressed by Darcy's law is taken into account in the HAM model. Indeed, the gradient of capillary suction is reduced to the water vapor pressure gradient and temperature gradient using Kelvin's law. Experimental validation of the proposed HAM model was undertaken in Ferroukhi et al. [9].

$$
\begin{aligned}
& C_{m_{i}}(\omega) \rho_{s_{i}} \frac{\partial P_{v}}{\partial t}=\operatorname{div}\left(k_{m_{i}}(\omega) \nabla P_{v}+k_{T_{i}} \nabla T+k_{p_{i}} \nabla P\right) \\
& C_{a_{i}} \frac{\partial P}{\partial t}=\operatorname{div}\left(k_{p_{i}} \nabla P\right) \\
& C_{p_{i}} \rho_{s_{i}} \frac{\partial T}{\partial t}=\operatorname{div}\left(\lambda_{i}(\omega) \nabla T+\alpha_{i} \nabla P_{v}+\gamma_{i} \nabla P\right)+L_{v} \rho_{s_{i}} \sigma_{i} C_{m_{i}}(\omega) \frac{\partial P_{v}}{\partial t}
\end{aligned}
$$

\subsection{HAM-BES co-simulation platform}

The co-simulation approach consists on coupling a dynamic building simulation tool (TRNSYS) with a coupled heat, air and moisture transfer model implemented in COMSOL. The coupling was carried out through MATLAB which represents an integrator tool ensuring the data exchange between TRNSYS and COMSOL. Indeed, TRNSYS is used here because it is widely used by scientific community and by engineers. In addition, its modular architecture is an advantage because it allows extending the modeling to a thermo-hydroaeraulic modeling. This is achievable by integrating ventilation types in simulations as Comis or Contam. For the hygrothermal transfer simulation in the building's walls, COMSOL was chosen in order to have an enough fine granularity simulation to describe the hygrothermal transfer in 2D and 3D especially in specific envelope components involving multidirectional flows such as thermal bridges.

The COMSOL/MATLAB connection is provided through the LiveLink interface [10]. Thus, the HAM model is defined in MATLAB and differential equations system is solved by the COMSOL solver. Concerning the TRNSYS/MATLAB link, it is established using the type 155 [11].

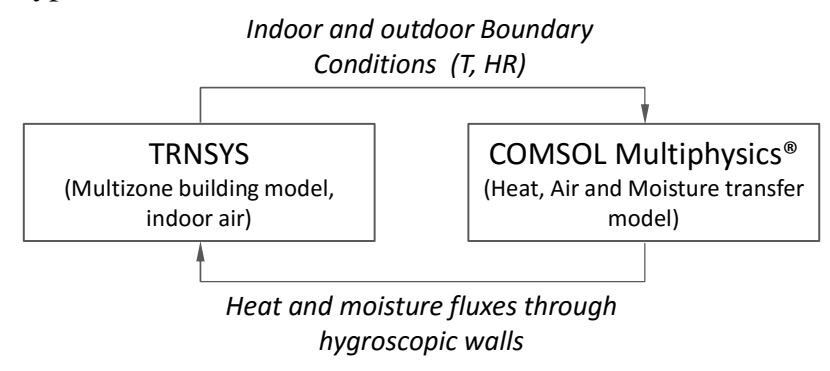

Fig. 7. Dynamic co-simulation approach between HAM (COMSOL) and BES (TRNSYS) models

In this approach, the control of the time step was provided by the BES model (TRNSYS). Consequently, the data exchange between the two coupled codes is made in the post-convergence to the current BES model time step during which the HAM model is solved in COMSOL with a finer time step. The diagram in Fig. 7 summarizes the temporal synchronization approach and 
data exchange strategy between the BES model in TRNSYS and the HAM model in COMSOL.

\subsection{Experimental investigation}

As expected previously, an experimental device adapted to study the hygrothermal response of buildings envelopes has been developed in this work. This experimental device is used to validate the proposed HAM-BES co-simulation approach.

The experimental validation was conducted by controlling relative humidity and temperature profiles for a steady state situation.

In this work, a monolayer chipboard wall with a thickness of $6.6 \mathrm{~cm}$ was used. The tested thickness envelope was taken enough small to optimize the test time, given the hygrothermal transfer kinetics and especially the moisture transfer which is relatively slow.

The test principle consists on submitting the envelope to two hygrothermal controlled environments. Each compartment was made of a plywood envelope (1.5 $\mathrm{cm})$ with an internal polystyrene insulation $(3 \mathrm{~cm})$. The inner surfaces of compartments walls were covered with sheet metal to avoid any moisture exchange with the indoor environment. The temperature is maintained for each ambiance with a thermostatic bath who feeds a copper heat exchange. For the relative humidity, a humidity generator was used. These apparatuses are equipped with deported sensors that allow a downstream hygrothermal control (ie in the two ambiences). Fig. 8 illustrate the developed experimental device.

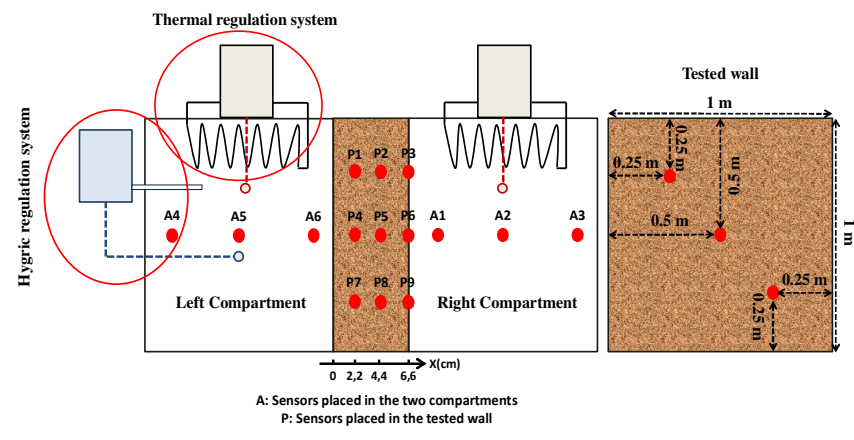

Fig. 8. Experimental device and sensors implementation

\subsection{Numerical simulation and validation}

In order to validate de developed HAM-BES platform, the numerical resolution obtained by this tool was compared to the experimental data provided from the experimentation previously presented.

It is noted that to perform the numerical simulation task, evidently, the initial and boundary conditions are identical to those of the experiment. For a better understanding of the presented experimental results, the placements of measurement points over the experimental device were illustrated in Fig. 8 .

The hygrothermal conditions imposed in the two controlled ambiences are represented in Fig.9.The numerical and experimental comparison of temperature and relative humidity distributions in the wall and the relative humidity in the compartment II are shown in Fig.10 and Fig. 11.Focusing on the temperature distribution through the wall, the results show good agreement between the experimental data and numerical simulation.

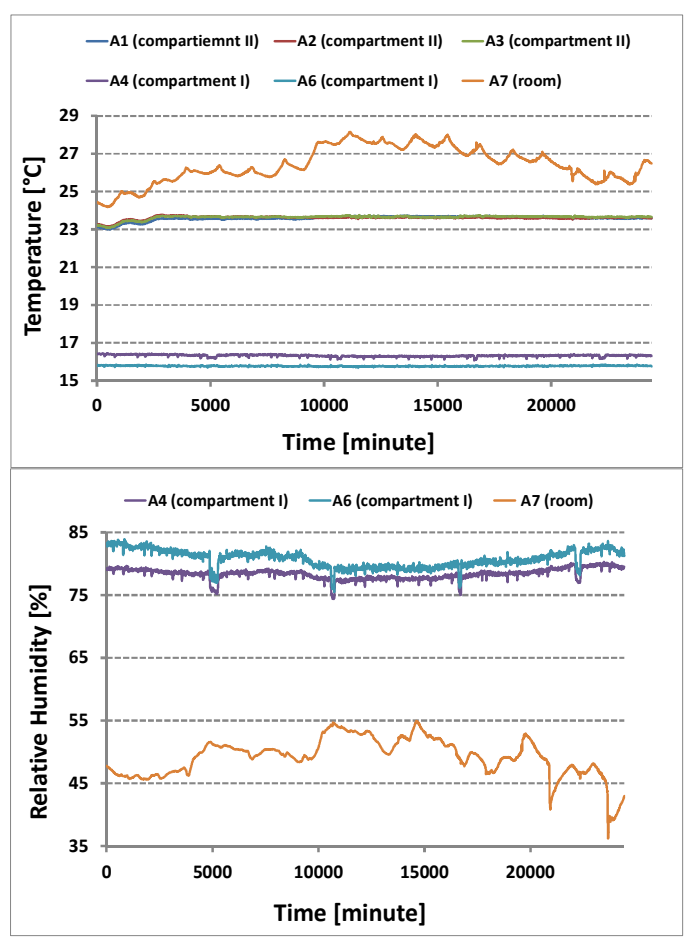

Fig.9. Imposed hygrothermal conditions in the two compartments

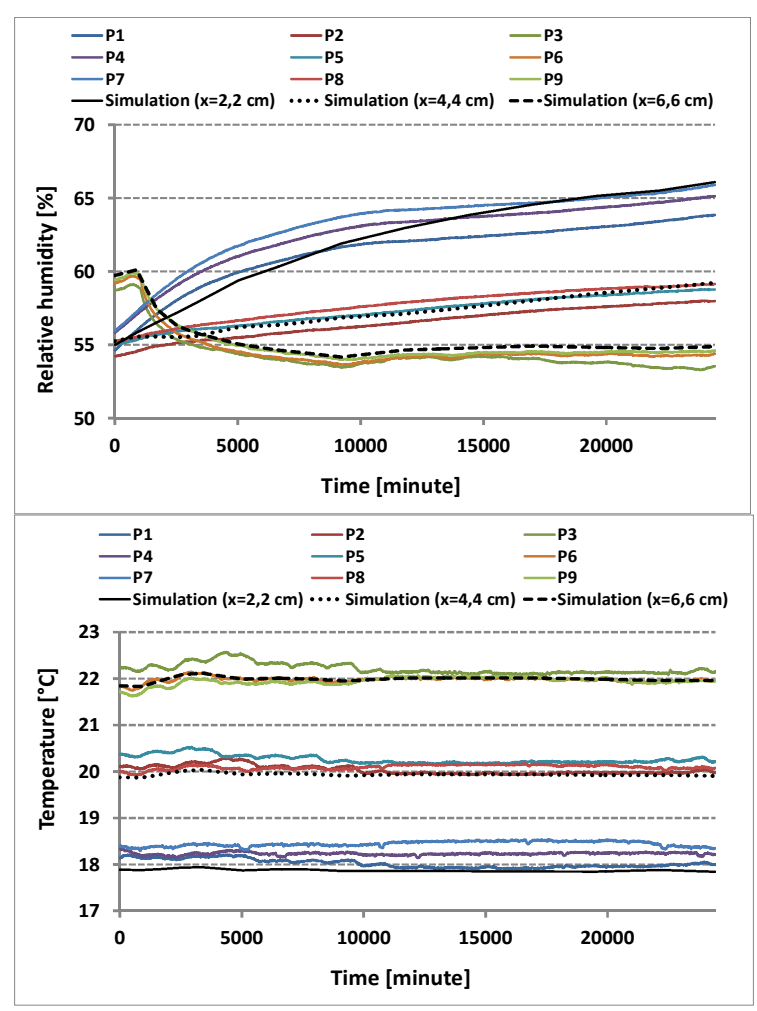

Fig.10. Comparison of numerical simulation with experimental data (temperature and relative humidity at different depths of the wall) 
The mean temperature deviation is about $0.2^{\circ} \mathrm{C}$ at a depth of $2.2 \mathrm{~cm}$. At $4.4 \mathrm{~cm}$ and $6.6 \mathrm{~cm}$, the mean deviations are $0.35^{\circ} \mathrm{C}$ and $0.30^{\circ} \mathrm{C}$ respectively. Concerning the comparison of the relative humidity distributions, a good agreement between predicted and measured data was observed. The mean variation reaches $2 \%, 1.5 \%$ and $1.3 \%$ at $2.2 \mathrm{~cm}, 4.4 \mathrm{~cm}$ and $6.6 \mathrm{~cm}$ of depth respectively. The same observation was noted by comparing the numerical and experimental data of the relative humidity variation in compartment II. The mean deviation over theexperimentation reaches $1.6 \%$ with a maximum of $2.7 \%$ (see Fig. 11).

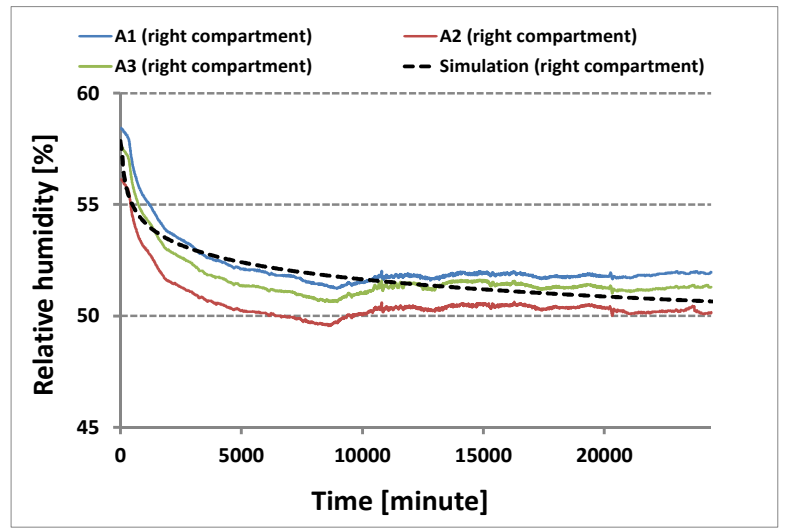

Fig.11.Comparison of numerical simulation with experimental data (Relative humidity variation in compartment II)

\section{Conclusions}

This paper is a contribution to the multi-scale study of the hygrothermal behavior of buildings to better understand the interactions between the different scales of building envelope materials. The microstructure of the materials was studied using X-ray tomography. This microscopic characterization highlights the complexity of the microstructure of porous building materials and their strong heterogeneities. The 3D reconstructions of the real material scanned with X-ray tomography at different resolutions show that hemp concrete has several scales of porosity, ranging from micro-porosity within the binder and hemp shiv to the inter-particle macro-porosity. In addition,thehygromorphic behavior under hydric solicitations was allowed to follow the deformations on a small scale to better understand the mechanisms of degradation of hemp concrete.

Then, the periodic homogenization method allowed to consider the heterogeneity and the complexity of the porous media and to describe their overall behavior on a large scale. It has been shown that macroscopic behavior of material is strongly dependent on the mechanisms acting at the levels of these constituents.

Finally, a coupled heat, air and moisture transfer model in multilayer walls was developed and examined. Further, an experimental device was designed to study the hygrothermal behavior of several configurations of multilayer porous building materials. Heat and moisture transfer evolutions in the samples were monitored over time, as well as good control of boundary conditions. Comparison of temperature and water vapor pressure profiles resulting from the numerical simulation with those obtained experimentally were undertaken. The results showed good agreement between predicted and measured data. These good results illustrate the advantages of the proposed hygrothermal transfer model, with accessible input data, evaluable experimentally with standardized tests.

Acknowledgments. This work was funded by the Region Aquitaine, Limousin, Poitou-Charentes Region through European and National Program CPER-FEDER "Bâtiment Durable 2015-2020".

\section{References}

1. K. Abahri, R. Belarbi, E.H. Chady, Caractérisation macro-hydrique des matériaux biosourcès, Rencontres Universitaires de Génie Civil. (2015).

2. S. Amziane, F. Collet, eds., Bio-aggregates Based Building Materials, Springer Netherlands, Dordrecht, (2017).

3. C. El Hachem, P. Ye, K. Abahri, R. Bennacer, Fiber's hygromorphic effect on thermal conductivity of wooden fibrous insulation characterized by X-ray tomography, Construction and Building Materials. 758-765. 150 (2017).

4. T. Taillandier-Thomas, S. Roux, T.F. Morgeneyer, F. Hild, Localized strain field measurement on laminography data with mechanical regularization, Nuclear Instruments and Methods in Physics Research Section B: Beam Interactions with Materials and Atoms. 70-79. 324 (2014).

5. F. Bennai, K. Abahri, R. Belarbi, A. Tahakourt, Periodic homogenization for heat, air, and moisture transfer of porous building materials, Numerical Heat Transfer,Part B: Fundamentals. 420-440. 70 (2016).

6. F. Bennai, K. Abahri, R. Belarbi, A. Tahakourt, Convective and conductive thermal homogenization for nonsturated porous building materials: Application on the thermal conductivity tensor, Thermal Science. (2016).

7. B. Remki, K. Abahri, M. Tahlaiti, R. Belarbi, Hygrothermal transfer in wood drying under the atmospheric pressure gradient, International Journal of Thermal Sciences. 57 (2012) 135-141.

8. Luikov, A. V, Heat and mass transfer in capillary porous bodies, PergamonLond. (1966).

9. M.Y. Ferroukhi, K. Abahri, R. Belarbi, K. Limam, A. Nouviaire, Experimental validation of coupled heat, air and moisture transfer modeling in multilayer building components, Heat and Mass Transfer. 2257-2269. 52 (2016).

10. Comsol Multiphysics User's Guide, ComsolMultiphysics User's Guide. (2012).

11. Solar Energy Laboratory, TRANSSOLAR Energietechnik GmbH, CSTB - Centre Scientifique et Technique du Bâtiment, et TESS - Thermal Energy Systems Specialists, TRNSYS 17 a TRaNsientSYstem Simulation program, vol. A3. (2010). 